Aus dem Institut zur Erforschung der Infektionskrankheiten in Himeji (Japan).

\title{
Antiluetin, ein neues Mittel der Kombinationstherapie.
}

Von Generalarzt Dr. M. Tsuzuki.

Die Kombinationstherapie geht von der Wahrnehmung aus, da $B$ verschiedene Substanzen in Kombination miteinander viel intensiver, ja sogar direkt heilend wirken können. Es ist daher im Hinblick auf diese Tatsache die Kenntnis der Kombinationsmöglichkeiten der verschiedenen Substanzen von großer Wichtigkeit. Im Prinzip besteht die Kombinationstherapie darin, die Erreger an mehreren Stellen zugleich anzugreifen und so zu bewirken, daß die einzelnen Substanzen, von denen jede für sich keine genügende Wirkung besitzt, vereint einen vollen Erfolg herbeiführen.

Die Kombinationstherapie ist für Trypanosomen zuerst von $\mathrm{La}$. veran, Mesnil, später von Wendelstadt, Moore, Nierenstein und Todd, Laveran und Thiroux, Friedberger, Löffler, Broden und Brodhain u. a. m. studiert und angewandt worden.

In meiner früheren Arbeit ${ }^{1}$ ), die ich während meines Aufenthaltes in Bern auf Anregung und unter Leitung von Herrn Prof. Kolle ausführte, habe ich die Wirkung von Arzneigemischen auf Trypanosomen am jebenden, infizierten Tiere untersucht und $\mathrm{kam}$ dabei $\mathrm{zu}$ folgenden Schlußfolgerungen:

„1. Die Kombination mehrerer chemotherapeutisch wirksamer Substanzen bei Behandlung der experimentellen Nagana-Trypanosomeninfektionen ergibt mit kleineren Mengen der einzelnen Heilmittel im Hinblick auf die Therapia sterilisans magna eine bessere Wirkung und einen sichereren Heileffekt als mit größeren Dosen der einzelnen Komponenten der Arzneimittel.

2. Die Kombination von Mitteln aus ein und derselben chemischen Gruppe gibt ungünstigere Resultate bezüglich des Heileffekts als die Kombination von Mitteln aus verschiedenen, chemisch weniger verwandten Gruppen.

3. Die Kombination mehrerer Substanzen mit verschiedenen An. griffspunkten im Sinne der Ehrlichschen Distributionsgesetze de Arzneimittel und Gifte, sowie in Analogie der Wirkung von Narcotiça nach Bürgi ermöglicht es, sterilisierende Arzneigemische herzustellen, die für den Gesamtorganismus relativ ungiftig sind, verglichen mit der toxischen Wirkung der sterilisierenden Dosis der einzelnen Medikamente."

1) Zeitschrift für Hygiene, Bd. 68, S. 364. 
Nach meiner Rückkehr nach Japan versuchte ich folgende Präparate gegen Syphilisspirochäten:

1. Stibio-Kali-tartaricum.

2. Antimonyl-anilintartrat.

3. Antimonyl-ammoniumtartrat.

Die Versuche wurden an Kaninchen vorgenommen, die nach den Methoden von Ossola, Truffi und Mezincescu mit syphilitischem Material infiziert wurden.

Die folgenden Tabellen demonstrieren kurz einige meiner Heilversuche bei Scrotumsyphilis der Kaninchen mit den oben genannten Präparaten.

\section{Tabelle 1.}

Resultat der Heilversuche bei Scrotumsyphilis mit StibioKali tartaricum.

\begin{tabular}{|c|c|c|c|c|}
\hline \multirow{2}{*}{ 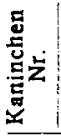 } & \multirow{2}{*}{$\begin{array}{l}\text { Krankheitszustand } \\
\text { und Spirochatten- } \\
\text { befund zur Zeit } \\
\text { der Behandlung }\end{array}$} & \multirow{2}{*}{\begin{tabular}{|c|} 
Behandlung: \\
Einmalige in- \\
travenose ohohr- \\
venee Injektion \\
Dosis pro kg
\end{tabular}} & \multicolumn{2}{|c|}{ Nach der Behandlung } \\
\hline & & & Spirochätenbefund & Resultate \\
\hline 1 & $\begin{array}{l}\text { Rechts groBer Schan. } \\
\text { ker mit vielen Spi- } \\
\text { rochäten }\end{array}$ & 0,005 & $\begin{array}{l}\text { Nach } 24 \text { Std. Spiro- } \\
\text { chäten weniger } \\
\text { zahlreich }\end{array}$ & 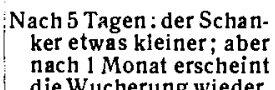 \\
\hline 2 & $\begin{array}{l}\text { Rechts u. links zlem. } \\
\text { lich groBer Schan- } \\
\text { ker mit vielen Spi. }\end{array}$ & 0,015 & do. & $\begin{array}{l}\text { die Wucherung wieder. } \\
\text { Nach } 8 \text { Tagen: Schanker } \\
\text { viel besser, aber nach } \\
\text { 3 Wochen Wucherung } \\
\text { wie frijer }\end{array}$ \\
\hline 3 & 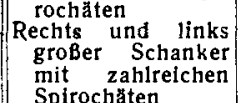 & 0,02 & do. & $\begin{array}{l}\text { wie früher. } \\
\text { Nach } 10 \text { Tagen: Schan- } \\
\text { ker gut geheilt. noch } \\
\text { nach } 6 \text { Monaten rezi- } \\
\text { divfrei. Monate }\end{array}$ \\
\hline 4 & $\begin{array}{l}\text { Links grober Schan- } \\
\text { ker mit vielen Spi- } \\
\text { rochâten }\end{array}$ & 0,025 & do. & Nach 26 Stunden Tod. \\
\hline
\end{tabular}

Tabelle 2.

Resultat der Heilversuche bei Scrotumsyphilis mit Antimonylanilintartrat.

\begin{tabular}{|c|c|c|c|c|}
\hline \multirow{2}{*}{ 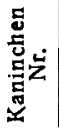 } & \multirow{2}{*}{$\begin{array}{l}\text { Krankheitszustand } \\
\text { und Spirochaten- } \\
\text { befund zur Zeit } \\
\text { der Behandlung }\end{array}$} & \multirow{2}{*}{$\begin{array}{l}\text { Behandlung: } \\
\text { Einmalige in: } \\
\text { travenōe (Ohr- } \\
\text { vene) Injekthon } \\
\text { Dosis pro kg }\end{array}$} & \multicolumn{2}{|c|}{ Nach der Behandlung } \\
\hline & & & Spirochătenbefund & Resultate \\
\hline 12 & $\begin{array}{l}\text { Rechts und links } \\
\text { groBer Schanker } \\
\text { mit zahlreichen }\end{array}$ & 0,005 & $\begin{array}{l}\text { Nach } 24 \text { Std. noch } \\
\text { viele Spirochăten }\end{array}$ & $\begin{array}{l}\text { Die Scrotumsyphilis un- } \\
\text { verändert; nach zwei } \\
\text { Monaten Tod durch }\end{array}$ \\
\hline 13 & $\begin{array}{l}\text { Rechts und links } \\
\text { grober Schanker } \\
\text { mit zahleichen } \\
\text { Spirochăten }\end{array}$ & 0,01 & $\begin{array}{l}\text { Nach } 24 \text { Std. Spiro- } \\
\text { chäten weniger } \\
\text { zahlreich }\end{array}$ & $\begin{array}{l}\text { Nach } 5 \text { Tagen Schanker } \\
\text { etwas besser ; aber } \\
\text { nach zwei Wochen } \\
\text { Wiederaftreten der }\end{array}$ \\
\hline 14 & $\begin{array}{l}\text { Rechts groBer Schan- } \\
\text { ker mit vielen Spi- } \\
\text { rochăten }\end{array}$ & 0.015 & do. & $\begin{array}{l}\text { Nach 5 Tagen. Schanker } \\
\text { in Heilung; aber nach } \\
\text { einem Monat Rezidiv. }\end{array}$ \\
\hline 15 & $\begin{array}{l}\text { Rechts und links } \\
\text { grober Schanker } \\
\text { mit vielen Spiro- } \\
\text { chäten }\end{array}$ & 0,02 & do. & $\begin{array}{l}\text { Nach } 6 \text { Tagen Schanker } \\
\text { gut geteilt; nach fünf } \\
\text { Monaten rezidivfrei. } \\
\text { ton }\end{array}$ \\
\hline 16 & $\begin{array}{l}\text { Rechts und links } \\
\text { grooer Schanker } \\
\text { mit vielen Spiro- } \\
\text { chätten }\end{array}$ & 0,025 & do. & Nach 48 Stunden Tod. \\
\hline
\end{tabular}

Tabelle 3.

Resultat der Heilversuche bei Scrotumsyphilis mit Antimonylammoniumtartrat.

\begin{tabular}{|c|c|c|c|c|}
\hline \multirow{2}{*}{ 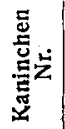 } & \multirow{2}{*}{$\begin{array}{l}\text { Krankheitszustand } \\
\text { und Spirochăten- } \\
\text { befund zur Zeit } \\
\text { der Behandlung }\end{array}$} & \multirow{2}{*}{\begin{tabular}{|l} 
Behandlung: \\
Einmalige in- \\
travenose olh- \\
vene) Injektion \\
Dosis pro kg \\
\end{tabular}} & \multicolumn{2}{|c|}{ Nach der Behandlung } \\
\hline & & & Spirochätenbefund & Resultate \\
\hline 22 & $\begin{array}{l}\text { RechtsgroBer Schan- } \\
\text { ker mit vielen Spi- } \\
\text { rochăteri }\end{array}$ & 0,005 & $\begin{array}{l}\text { Nach } 24 \text { Std. Spiro- } \\
\text { chäten weniger } \\
\text { zahlreich. }\end{array}$ & $\begin{array}{l}\text { Nach einer Woche } \\
\text { Schanker etwaskleiner, } \\
\text { aber nach einem Monat } \\
\text { erscheint die Wuche- }\end{array}$ \\
\hline 23 & $\begin{array}{l}\text { Rechts und links } \\
\text { grober Schanker } \\
\text { mit vielen Spiro- } \\
\text { chăten }\end{array}$ & 0.01 & do. & $\begin{array}{l}\text { rung wieder. } \\
\text { Nach 10 Tagen Schan- } \\
\text { ker etwas besser, aber } \\
\text { nach einem Monat } \\
\text { Wiederauftreten der der }\end{array}$ \\
\hline 24 & $\begin{array}{l}\text { Rechts und links } \\
\text { groBer Schanker } \\
\text { mit vielen Spiro- } \\
\text { chăten }\end{array}$ & 0,015 & do. & $\begin{array}{l}\text { Spirochäten. } \\
\text { Nach } 10 \text { Tagen Schan- } \\
\text { ker gut geheilt, nach } \\
6 \text { Monaten noch rezi- } \\
\text { divfrei. }\end{array}$ \\
\hline 25 & $\begin{array}{l}\text { Links groBer Schan- } \\
\text { ker mit vielen Spi- } \\
\text { rochäten }\end{array}$ & 0,02 & do. & $\begin{array}{l}\text { Nach } 12 \text { Tagen Schan- } \\
\text { ket gut geheilt. nach } \\
6 \text { Monaten noch rezi- }\end{array}$ \\
\hline 27 & $\begin{array}{l}\text { Rechts und links } \\
\text { grober Schanker } \\
\text { mit vielen Spiro- } \\
\text { chăten }\end{array}$ & 0,003 & do. & $\begin{array}{l}\text { divtreli. } \\
\text { Nach } 48 \text { Stunden Tod. }\end{array}$ \\
\hline
\end{tabular}

Aus diesen Tabellen ergibt sich, daß das Antimonyl-ammoniumtartrat vom Kaninchen gut vertragen wird und eine verhältnismäßig günstige Wirkung aufweist. Ich habe diese
Substanzen auch bei Syphilitikern subkutan angewandt (pro $50 \mathrm{~kg}$ Körpergewicht $0,75 \mathrm{~g}$ ), jedoch ohne jeden Erfolg. Schon geringe Dosen von $0,025-0,05 \mathrm{~g}$ lösten starke örtliche Reaktionen bei den behandelten Personen aus. Die Patienten empfanden an der Injektionsstelle starken Schmerz. In einigen Fällen kam es sogar zur Vereiterung. Wegen der geringen Heilerfolge bei Syphilitikern habe ich davon Abstand genommen, diese Substanzen weiterhin zu verwenden.

Bei meinen folgenden Versuchen war ich bemüht, mit einem Mittel zu arbeiten, dessen organotrope Eigenschaften vermindert, die parasitotropen dagegen erhöht waren; d. h. das Mittel soll nur für die in den Körper eingedrungenen Mikroorganismen, nicht aber für den infizierten Körper chemische Verwandtschaft besitzen. Die Chemotherapie ist in der neuesten Zeit durch zielbewußte systematische Untersuchungen zu Substanzen gelangt, die ,sich dem Ideal eines parasitotropen, aber nicht organotropen Spezifikums immer mehr nähern, weil man in den gleichen Medikamenten eine ganze Gruppe von sehr wirksamen spezifischen Arzneien zu finden hofft".

Die neue Substanz, die ich mir herstellte und die ich zu meinen Untersuchungen verwendete, ist das BitartratoKalium-ammonium-antimonoxyd.

$\left(\mathrm{Sb} \mathrm{O}\left(\mathrm{C}_{4} \mathrm{H}_{4} \mathrm{O}_{6}\right)_{2}\right) \mathrm{K}\left(\mathrm{NH}_{4}\right)_{2} \mathrm{H}_{2} \mathrm{O}$.

Dieses Salz habe jch „Antiluetin" genannt und auch unter diesem Namen in den Handel gebracht.

Tabelle 4.

Resultat der Heilversuche bei Scrotumsyphilis mit Antiluetin.

\begin{tabular}{|c|c|c|c|c|}
\hline 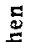 & & Behandlung: & \multicolumn{2}{|c|}{ Nach der Behandlung } \\
\hline z & $\begin{array}{l}\text { befund zur Zeit } \\
\text { der Behandlung }\end{array}$ & $\begin{array}{l}\text { travenosse (Ohr- } \\
\text { vene) Injektion } \\
\text { Dosis pro kg }\end{array}$ & Spirochätenbefund & Resultate \\
\hline 37 & $\begin{array}{l}\text { Links groBer Schan- } \\
\text { ker m. zahlreichen } \\
\text { Spirochăten }\end{array}$ & 0,01 & $\begin{array}{l}\text { Nach } 24 \text { Std. Spiro- } \\
\text { chäten weniger } \\
\text { zahlreich }\end{array}$ & $\begin{array}{l}\text { Nach } 8 \text { Tagen Schanker } \\
\text { etwas kleiner; nach } \\
\text { 1 Monat erscheint die }\end{array}$ \\
\hline 40 & $\begin{array}{l}\text { Rechts und links } \\
\text { groBer Schanker } \\
\text { mit vielen Spiro- } \\
\text { chäten }\end{array}$ & 0,015 & de & $\begin{array}{l}\text { Nach } 8 \text { Tagen Schanker } \\
\text { verschwunden; nach } \\
6 \text { Monaten noch rezi- } \\
\text { divfrei. }\end{array}$ \\
\hline 41 & $\begin{array}{l}\text { Rechts groBer Schan- } \\
\text { ker mit vielen Spi. } \\
\text { rochaten }\end{array}$ & 0,02 & do. & $\begin{array}{l}\text { Nach } 10 \text { Tagen Schanker } \\
\text { verschwunden; nach } \\
6 \text { Monaten noch rezi- }\end{array}$ \\
\hline 45 & $\begin{array}{l}\text { Links groBer Schan- } \\
\text { ker mit vielen Spi- } \\
\text { rochäten }\end{array}$ & 0.025 & do. & $\begin{array}{l}\text { Nach } 7 \text { Tagen Schanker } \\
\text { verschwunden; nach } \\
6 \text { Monaten noch rezi- }\end{array}$ \\
\hline 46 & $\mid \begin{array}{c}\text { Rechts und links } \\
\text { Schanker m. vielen } \\
\text { Spirochäten }\end{array}$ & 0,03 & do. & Nach 76 Stunden Tod. \\
\hline
\end{tabular}

Kurz seien hier die chemischen Reaktionen des "Antiluetins" angegeben:

1. Eine kleine Probe dieses Salzes gibt mit Chlorwasserstoffsäure in der Flamme ein stark violettes Licht infolge seines Kaliumgehaltes.

2. Kochen des Salzes im Reagenzglase mit $\mathrm{NaOH}-\mathrm{Lösung}$ entwickelt starken Geruch nach Ammoniak infolge seines Gehaltes an $\mathrm{NH}_{\mathbf{q}}$-Gruppen.

3. Das "Antiluetin" gibt mit einer $5 \%$ igen Ammoniaklósung einen Niederschlag von Antimonhydroxyd. Diese Probe ist aber nicht ausreichend. Besser versetzt man daher die HCL-saure Lösung des Salzes mit Zink, wodurch das Antimon als solches in schwarzen Flocken aus fällt. Gleichzeitig entwejcht ein Teil des Antimons als flüchtiges $\mathrm{SbH}_{3}$, das Silbernitratpapier schwärzt.

Im folgenden mehr theoretischen Teil möchte ich die Frage eingehend erörtern, wie diese neue Substanz, die chemisch so ganz anders konstituiert ist als diejenigen Substanzen, von denen Ehrlich ausgegangen ist, mit den Grundsätzen seiner Seitenkettentheorie in Uebereinstimmung zu bringen ist.

Eine Vergleichung des „Antiluetins" mit dem bekannten Dihydroxydiaminoarsenobenzol Ehrlichs:<smiles>NC1CCC(C=CC2CC(O)CC2N)C1</smiles>

Auf die chemische Konstitution und die damit in Beziehung stehende pharmakodynamische Wirkung mag deshalb hier eingegangen werden. Stellen wir uns die verschiedenen Momente, die für die spirillozide Funk. tion bei Salvarsan von Einfluß sind, soweit dies bis jetzt wenigstens möglich ist, einmal übersichtlich zusammen, so ergibt sich dabei folgendes:

1. Es kann nach Ehrlich als sicher angesehen werden, daß die „abtötende Funktion" dieses komplizierten Moleküls auf den in der 
Arsenogruppe enthaltenen dreiwertigen Arsenrest $(-\mathrm{As}=\mathrm{As} \rightarrow$ zurückzuführen ist.

Was die Verbindungen des fünfwertigen Arsens betrifft, so sind sie zwar weniger giftig, aber es kann - bei Mäusen wenigstens - eine wirkliche Sterilisation nur mit sehr hohen Dosen erreicht werden, die hart an der Grenze der Dosis tolerata stehen und zu chronischen Vergiftungen führen. Große Erfolge in chemotherapeutischer Hinsicht sind deshalb von ihnen nicht zu erwarten.

Bei der Prüfung der dreiwertigen Arsenderivate hat sich ferner herausgestellt, daß das dem Salvarsan entsprechende Arsenoxyd in bezug auf die spirillozide Fähigkeit dem Salvarsan, wenn nicht überlegen, so doch wenigstens ebenbürtig ist. Allerdings ist die Toxizität der Arsenoxydgruppe $(-\mathrm{As}=0)$ viel höher als diejenige der Arsenoverbindung, was die praktische Anwendbarkeit in Frage stellt.

Die Bemühungen, Substanzen zu finden, die für den Menschen noch unschädlicher sind als Salvarsan, ohne die spirillozide Wirkung zu vermindern, haben seit Ehrlichs Entdeckung nicht nachgelassen. Es ist vorauszusehen, daß geeignetere komplexe Verbindungen noch aufgefunden werden. Hier konnte kein Gedanke näher liegen, als die giftige $(-\mathrm{As}=0)$ Arsengruppe gegen die entsprechende, weniger giftige Gruppe des Antimons ( $-\mathrm{S} b=0$ ) umzutauschen.

2. Nach Ehrlich tritt nur dort Bindung der chemischen Substanz mit einer Spirochätenzelle ein, wo für diese die entsprechenden haptophoren Gruppen in der Zelle vorhanden sind.

Bei Salvarsan kommen die OH-Gruppen in $\beta$-Stellung dafür in Betracht. Sie kommen für die spirillozide Wirkung ausschließlich in Betracht, und durch sie allein wird die Verankerung an die haptophore Gruppe bedingt.

Der Gedanke liegt auch hier nahe, statt des Benzolringes mit den OH-Gruppen den Weinsäurerest zu verwenden, eine Substanz, die ja in der organischen Chemie bereits seit $P$ asteurs Zeiten dadurch bekannt ist, $\mathrm{da} B$ sie im Lebensproze $B$ besonderer Bakteriengruppen sogar die Stelle eines Nahrungsstoffes einnehmen kann, worauf sich ja spezielle Methoden zur Trennung optisch aktiver Substanzen für stereochemische Zwecke gegründet haben. Jedenfalls stehen organische Molekülreste aus der Reihe der aliphatischen Verbindungen, wie die Weinsäuræ z. B., in engerer Verwandtschaft zu dem Leben der Parasiten oder der niederen Organismen als der aromatische Benzolring, der mehr für Produkte aus dem LebensprozeB der höheren Organismen charakteristisch ist.

Auch die Weinsäuregruppe $\left(\mathrm{C}_{4} \mathrm{H}_{4} \mathrm{O}_{6}\right)$ würde, ähnlich wie Salvarsan, zwei OH-Gruppen enthalten, die für haptophore Gruppen der Zelle in Betracht kämen. Es sind diejenigen, die in folgender Formel ein. geklammert sind:

$$
<\begin{aligned}
& \mathrm{O}-\mathrm{CO}-\mathrm{CH}^{\prime} \\
& \text { O. }-\mathrm{CO}-\mathrm{CH} \mathrm{Y}_{(\mathrm{OH})}
\end{aligned}
$$

Dies sind die Argumente, die zur versuchsweisen Anwendung des Stibio-Kali-tartaricum berechtigten und auf Grund der Ehrlichschen Seitenkettentheorie guten Erfolg versprachen. Hier seien die Formeln der angewendeten Antimonpräparate wiedergegeben.

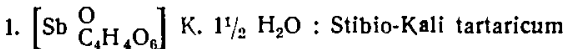

$$
\begin{aligned}
& \text { 2. }\left[\mathrm{Sb}_{\mathrm{C}_{4} \mathrm{H}_{4} \mathrm{O}_{6}}^{\mathrm{O}}\right] \mathrm{NH}_{2}{ }_{\mathrm{C}_{6} \mathrm{H}_{2}}^{\mathrm{H}} \mathrm{H}_{2} \mathrm{O} \text { : Antimonyl-anilintartrat. } \\
& \text { 3. }\left[\mathrm{Sb} \mathrm{C}_{4} \mathrm{H}_{4} \mathrm{O}_{6}\right] \mathrm{NH}_{4} 11 / 2 \mathrm{H}_{2} \mathrm{O}: \text { Antimonyl-ammoniumtartrat. }
\end{aligned}
$$

Um die chemotherapeutische Wirkung dieser Substanzen richtig zu verstehen, ist es unbedingt nötig, sich mit der chemischen Natur dieser Körper etwas bekannt zu machen. Es dürfte dies nicht überflüssig sein, da die diesbezüglichen Anschauungen nur im engsten Kreise der Chemotherapeutiker eingehend gewürdigt werden. Denn mit der Chemie der sogenannten „Koordinationsverbindungen" haben sich bisher nur einige Spezialforscher eingehender beschäftigt. An erster Stelle steht der Name A. Werners, ${ }^{1}$ ) der auch den Begriff der Koordination in die Chemie eingeführt und so den Begriff der Valenz erweitert und ausgebildet hat.

Nach ihm sind die Koordinationsverbindungen so konstituiert, daß immer - in normalen Fällen - - sechs Atomgruppen mit dem Metallatom (Antimon) direkt verbunden sind, oder, etwas anders ausgedrückt, bei ,koordinativ gesättigten Verbindungen" immer sechs Raumstellen um das Metallatom herum von fremden Atomen in Beschlag genommen werden.

Betrachten wir daraufhin unsere drei Verbindungen, so sehen wir, daß die Koordinationszahl den Wert sechs nicht erreicht, sondern nur vier beträgt, indem freie 0 -Atome immer zwei Raum- oder Koordinationsstellen einnehmen, während bereits mit anderen Gruppen in Verbindung stehende $\mathrm{O}$-Atome nur je eine Stelle beanspruchen. Ein an-

1) Neuere Anschauungen auf dem Gebiete der anorganischen Chemie. 2. Auflage, 1909. schauliches Bild von diesen Verhältnissen gibt die folgende Koordinationsformel für Stibio-Kali tartaricum:

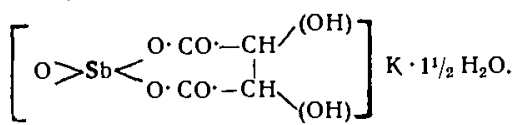

Auf die Konsequenzen dieser Erscheinung werden wir noch einmal zurückkommen.

Wenngleich die „parasitotrope Funktion" dieser Substanzen sich schon durch das erklärt, was innerhalb der eckigen Klammern, oder - chemisch gesprochen - durch die toxophore Gruppe des Antimonoxydrestes und den bakteriophilen Weinsäurerest mit seinen zwei haptophoren $O H$-Gruppen vorhanden ist, so ist doch auch ersichtlich, daß das, was in der äußeren Sphäre des Moleküls, außerhalb der eckigen Klammer, enthalten, von Einfluß sein wird auf die spirillozide Funktion dieser Substanzen.

Die Wirkungsweise dieser außenstehenden Gruppen erscheint etwas rätselhaft. Immerhin ist sie wohl in Analogie zu bringen zu der Wirkung der $\mathrm{NH}_{2}$-Gruppen im ,Salvarsan“. Ehrlich ist der Ansicht, daß die Nachbarstellung dieser Aminogruppen zum OH eine wesentliche Erhöhung der Wirkung dieser letzteren hervorzurufen imstande ist. Derartige Fälle sind ja dem organischen Chemiker so geläufig, daß nichts Erzwungenes in dieser Erklärung gefunden werden kann. Aehnlich wie in Salvarsan die $\mathrm{NH}_{2}$-Gruppen als die ,wirkungserhöhenden" Gruppen aufgefaßt werden müssen, so können in den drei oben genannten Salzen die außenstehenden Reste je nachdem als "wirkungserhöhende" oder "wirkungshemmende" betrachtet werden.

Die Theorie A. Werners lehrt, daß solche Reste mehr oder weniger fest mit dem Molekülkomplex verbunden sind. Der Grad, die Stärke der Verkettung ist von Substanz zu Substanz verschieden und hängt auch von der Dissoziation der Salze in wäBriger Lösung ab. Es ist möglich, daß sie, wenn sie fest damit verbunden sind, die Seitenketten des Weinsäurerestes, die ,haptophoren“"Gruppen des Komplexes verdecken und diesen so vor einer Zerstörung durch die Spirochäten, die sich sonst mit Gier darauf stürzen würden, zu schützen vermögen. Es ist dann auch verständlich, daß bei loser Verbindung der Außenradikale mit dem Komplex diese sich frei in der Lösung herumbewegen, unabhängig vom Komplex, und so der Komplex den Angriffen der entsprechenden Rezeptoren schneller unterliegt, weil eben die Seitenketten in diesem Falle ungeschützt sind.

Sei dem, wie es will. Offenbar wird die Dissoziation in diesem Proze $B$ auch eine Rolle spielen, um so mehr, als diese Substanzen immer in einer gewissen Verdünnung, also in stark dissoziativem Zustande, dem Körper einverleibt werden. Es würden selbstverständlich auch genauere physikalisch-chemische Untersuchungen nötig sein, wenn man diesen Fragen genau auf den Grund gehen wollte.

Wir haben oben gesehen, daB die drei Salze die maximale Koordinationszahl sechs nicht erreicht haben. Sie beträgt nur vier. Deshalb sind diese als "koordinativ ungesättigte Verbindungen" zu betrachten. Und als solche werden sie das Bestreben haben, durch Aufnahme weiterer Molekülgruppen in gesättigte Verbindungen überzugehen. Und gerade diese Tendenz zur Absättigung läßt den Verdacht in uns aufkommen, daß die ausgesprochene Organotropie dieser Substanzen darin ihren Grund haben könnte und daß diese ungesättigten Moleküle allzusehr dahin neigten, sich mit lebenswichtigen Organozeptoren unseres Körpers zu verankern, sich an ibnen abzusättigen.

Es ist aber, schon theoretisch, die Möglichkeit gegeben, dies künstlich zu verhindern, und zwar durch Ueberführung dieser ungesättigten Verbindungen in ,koordinativ gesättigte“. Die Praxis hat denn auch nach vielen Versuchen gezeigt, daß eine chemische Absättigung mit Ammoniumtartrat, insbesondere beim Stibio-Kalium tartaricum zu einem vollen Erfolg führt.

Auf diese Weise sind wir zur Herstellung des Bitartrato-Kaliumammonium-antimonoxyd gelangt, dem offenbar die folgende Konstitutionsformel zukommen dïrfte:

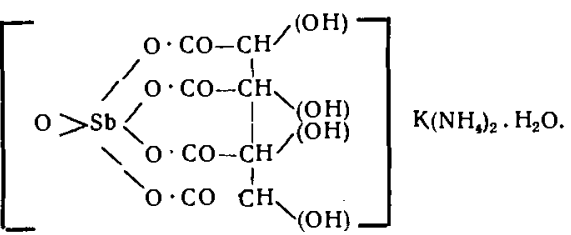

Diese Formel erklärt mit Leichtigkeit die spirillozide Wirkung, uf Grund der Ehrlich schen Seitenkettentheorie, wie sie für rein organische Verbindungen entwickelt wurde. Es wird auch verständlich, warum durch diese Absättigung nicht nur die Organotropie bedeutend reduziert, sondern auch die parasitotrope Wirkung erhöht wird, vielleicht daß durch die Einführung eines neuen Weinsäurerestes die Zahl der haptophoren Gruppen um zwei weitere vermehrt wird. 